\title{
Static quark-antiquark potential and Dirac eigenvector correlators
}

\author{
Erek Bilgici and Christof Gattringer \\ Institut für Physik, Unversität Graz \\ 8010 Graz, Austria
}

\begin{abstract}
We represent the Polyakov loop correlator as a spectral sum of correlators of eigenvectors of the lattice Dirac operator. This spectral representation is studied numerically using quenched $\mathrm{SU}(3)$ configurations below and above the deconfinement temperature. We analyze whether the individual Dirac eigenvector correlators differ in the confined and deconfined phases. The decay properties of the normalized Dirac eigenvector correlators turn out to be essentially identical in the two phases, but the amplitudes change. This change of the amplitudes shifts the relative contributions of the individual Dirac eigenvector correlators and is the driving mechanism for the transition from the confining static potential into the deconfining one.
\end{abstract}




\section{Introductory remarks}

Confinement of quarks is one of the key features of QCD. Of particular interest is the fact that at a critical temperature QCD undergoes a transition into a deconfined high temperature phase. Understanding the transition and characterizing QCD in the deconfined phase are widely discussed problems.

It is obvious, that confinement and deconfinement, respectively, must be manifest in the Dirac operator and its inverse, the quark propagator, since the quarks need to know whether they are confined or not. In a series of recent papers [1] - 7] the connection of quantities related to confinement, e.g., the expectation value of the Polyakov loop, to spectral sums of the Dirac operator was studied. Signatures of confinement in the Dirac spectrum and their change at the QCD transition were identified.

In this article we go beyond results for only the Dirac eigenvalues and study a spectral representation of the static quark anti-quark potential. Since this quantity depends on the relative spatial position of the static sources, its spectral representation contains also the eigenvectors of the Dirac operator. In particular the shape of the potential must reflect itself in spatial correlators of Dirac eigenvectors. Very little is known about such correlators of Dirac eigenvectors and we study these relations numerically using quenched lattice QCD configurations. We explore how the spectral signatures of Dirac eigenvalues and eigenvectors change at the deconfinement transition.

We remark at this point that very recently Synatschke, Wipf and Langfeld have studied correlators of spectral sums with a different, IR dominated spectral function [7]. Using the boundary condition technique of [1] they project to loops with the same transformation properties as the Polyakov loops which we use here. The continuum arguments given in [7] and the numerical study with $\mathrm{SU}(2)$ gauge configurations provide interesting complementary insight to the analysis presented here.

\section{Dirac operator and Polyakov loops}

In this section we repeat and refine the arguments [1] which lead to the connection of the Polyakov loop and its correlators to spectral sums for the Dirac operator.

The Polyakov loop at spatial position $\vec{x}$ is given by

$$
L(\vec{x})=\frac{1}{n} \operatorname{tr}_{c}\left[\prod_{t=1}^{N} U_{4}(\vec{x}, t)\right] .
$$

$U_{4}(\vec{x}, t)$ is the temporal component (=4-component) of the gauge variables 
$U_{\mu}(\vec{x}, t) \in \mathrm{SU}(n)$ and $\operatorname{tr}_{c}$ is the color trace. The rank $n$ of the gauge group in the fundamental representation appears in the overall normalization of the Polyakov loop. The product runs over all $N$ temporal links at a given spatial point $\vec{x}$. We work on a lattice of size $L^{3} \times N$ and for the gauge field use boundary conditions which are periodic in all four directions. We are interested in correlators of the Polyakov loop

$$
\left\langle L(\vec{x}) L^{*}(\vec{y})\right\rangle \sim A e^{-N V(|\vec{x}-\vec{y}|)},
$$

where * denotes complex conjugation and $V(|\vec{x}-\vec{y}|)$ is the static quark-antiquark potential.

The staggered Dirac operator at vanishing quark mass is given by (we set the lattice spacing to 1 )

$$
D(x, y)=\frac{1}{2} \sum_{\mu=1}^{4} \eta_{\mu}(x)\left[U_{\mu}(x) \delta_{x+\hat{\mu}, y}-U_{\mu}(x-\hat{\mu})^{\dagger} \delta_{x-\hat{\mu}, y}\right],
$$

where $x$ and $y$ are integer valued 4-vectors labeling the lattice sites and $\eta_{\mu}(x)=$ $(-1)^{x_{1}+\ldots+x_{\mu-1}}$ is the staggered sign function.

The terms of the staggered Dirac operator (3) connect nearest neighbors. When powers of $D$ are considered, these terms combine to chains of hops on the lattice. Along these chains products of the link variables $U_{\mu}(x)$ are collected. Taking the $m$-th power will give rise to chains of length $m$. We now consider the $N$-th power of $D$, where $N$ is the temporal extent of our lattice. Thus we will encounter chains with a length of $N$. Furthermore we set the two space-time arguments of $D$ to the same value, $y=x$, such that we pick up only closed chains, i.e., loops starting and ending at $x$. Among these are the loops where only hops in time direction occur such that they close around compact time. We obtain

$$
\begin{aligned}
& \operatorname{tr}_{c}\left[D^{N}(\vec{x}, t \mid \vec{x}, t)\right]=\text { trivial loops }+\frac{(-1)^{N\left(x_{1}+x_{2}+x_{3}\right)}}{2^{N}} \operatorname{tr}_{c} \prod_{s=1}^{N} U_{4}(\vec{x}, s) \\
&+\frac{(-1)^{N\left(x_{1}+x_{2}+x_{3}\right)}}{2^{N}} \operatorname{tr}_{c} \prod_{s=0}^{N-1} U_{4}(\vec{x}, N-s)^{\dagger} \\
&= \text { trivial loops } \\
&+\frac{1}{2^{N}} n L(\vec{x})+\frac{1}{2^{N}} n L^{*}(\vec{x})
\end{aligned}
$$

The factor $(-1)^{N\left(x_{1}+x_{2}+x_{3}\right)}$ comes from the products of the staggered sign factors $\eta_{4}(x)$. Here we use even $N$, such that this factor is equal to +1 . We stress that the forward and backward running Polyakov loops are the only ones that wind non-trivially around compact time. 
We now explore the fact that the Polyakov loops respond differently to a change of the boundary conditions compared to other, trivial (= non-winding) loops. We can change the temporal boundary condition of the Dirac operator by multiplying all temporal link variables at $t=N$ with some phase factor $z \in \mathbb{C},|z|=1: U_{4}(\vec{x}, N) \rightarrow z U_{4}(\vec{x}, N) \forall \vec{x}$. We evaluate the left-hand side of (4) using the Dirac operator in the transformed field, and denote the corresponding Dirac operator as $D_{r}$, where $r$ labels the boundary conditions we use. Actually we linearly combine three different boundary conditions $z_{r}$, $r=1,2,3$ to obtain:

$$
\begin{aligned}
& \frac{2^{N}}{n} \sum_{r=1}^{3} c_{r} \operatorname{tr}_{c}\left[D_{r}^{N}(\vec{x}, t \mid \vec{x}, t)\right]=L(\vec{x}) \times \sum_{r=1}^{3} c_{r} z_{r} \\
& +L^{*}(\vec{x}) \times \sum_{r=1}^{3} c_{r} z_{r}^{*}+\text { trivial loops } \times \sum_{r=1}^{3} c_{r} .
\end{aligned}
$$

The complex coefficients $c_{r}$ are determined by the requirement that the factors in front of the complex conjugate loop $L(\vec{x})^{*}$ and in front of the trivial loops vanish, while the factor in front of the Polyakov loop $L(\vec{x})$ is required to be equal to 1 . Here we use the following values for the $z_{r}$ and the $c_{r}$,

$$
z_{1}=1, z_{2}=e^{i 2 \pi / 3}, z_{3}=e^{-i 2 \pi / 3} \quad \text { and } \quad c_{r}=z_{r}^{*} / 3,
$$

which fulfill the above requirements. We conclude

$$
L(\vec{x})=\frac{2^{N}}{n N} \sum_{t=1}^{N} \sum_{r=1}^{3} z_{r}^{*} \operatorname{tr}_{c}\left[D_{r}^{N}(\vec{x}, t \mid \vec{x}, t)\right] .
$$

We have used the fact that the left-hand side is independent of $t$ and averaged the right-hand side over $t$.

\section{Spectral sum for the Polyakov loop correlator}

We now express the $N$-th power of the Dirac operator as a spectral sum. Let $\vec{v}^{(k)}(\vec{x}, t)$ be the eigenvector of $D$ with eigenvalue $\lambda^{(k)}$. Then

$$
D^{N}(\vec{x}, t \mid \vec{x}, t)_{c, c^{\prime}}=\sum_{k}\left(\lambda^{(k)}\right)^{N} \vec{v}^{(k)}(\vec{x}, t)_{c} \vec{v}^{(k)}(\vec{x}, t)_{c^{\prime}}^{*},
$$

where we have made explicit also the color indices $c, c^{\prime}$. The index $k$ runs over all eigenvalues of the Dirac operator. Inserting the spectral sum into (17) we find

$$
L(\vec{x})=\frac{2^{N}}{n} \sum_{k} \sum_{r=1}^{3} z_{r}^{*}\left(\lambda_{r}^{(k)}\right)^{N} \rho_{r}^{(k)}(\vec{x}),
$$




$$
\rho_{r}^{(k)}(\vec{x})=\frac{1}{N} \sum_{t=1}^{N} \sum_{c=1}^{n}\left|\vec{v}_{r}^{(k)}(\vec{x}, t)_{c}\right|^{2},
$$

where again $r$ labels the three different boundary conditions we use and the index $k$ runs over all eigenvalues.

The spectral sum may be simplified further, using the fact that the eigenvalues of the staggered Dirac operator are purely imaginary and come in complex conjugate pairs. The eigenvectors corresponding to these pairs are related to each other by multiplication with the staggered sign $(-1)^{x_{1}+x_{2}+x_{3}+x_{4}}$, which leaves the densities (10) invariant. Thus in the spectral formula (9) one needs to sum over only the eigenvalues with positive imaginary part (we indicate this with the superscript + attached to the summation symbol) and an extra factor of 2 appears. Thus we obtain for the Polyakov loop correlator

$$
L(\vec{x}) L^{*}(\vec{y})=\frac{4^{N+1}}{9 n^{2}} \sum_{k, l}^{+} \sum_{r, s=1}^{3} z_{r}^{*} z_{s}\left(\lambda_{r}^{(k)} \lambda_{s}^{(l)}\right)^{N} \rho_{r}^{(k)}(\vec{x}) \rho_{s}^{(l)}(\vec{y}) .
$$

We stress that the result (11) is an exact formula, in particular it holds for an individual gauge field configuration and not only in the ensemble average. Furthermore, all terms involved in our spectral representation are manifestly gauge invariant quantities.

Let us finally discuss the correlator after the vacuum expectation value $\langle.$. has been evaluated. In this case one can use the fact that the expectation value is invariant under spatial reflections, i.e., we can interchange $\vec{x}$ and $\vec{y}$. When using the choice (6) for the phases at the boundary one obtains a particularly simple form of the correlator

$$
\begin{aligned}
\left\langle L(\vec{x}) L^{*}(\vec{y})\right\rangle=\frac{4^{N+1}}{9 n^{2}} \sum_{k, l}+\left[\sum_{r=1}^{3}\langle\right. & \left.\left(\lambda_{r}^{(k)} \lambda_{r}^{(l)}\right)^{N} \rho_{r}^{(k)}(\vec{x}) \rho_{r}^{(l)}(\vec{y})\right\rangle \\
& \left.-\sum_{r<s}\left\langle\left(\lambda_{r}^{(k)} \lambda_{s}^{(l)}\right)^{N} \rho_{r}^{(k)}(\vec{x}) \rho_{s}^{(l)}(\vec{y})\right\rangle\right] .
\end{aligned}
$$

The final result is a sum of 6 terms, where the 3 correlators of the densities with equal boundary condition come with a plus sign, while the correlators with mixed boundary conditions are subtracted.

\section{Truncated spectral sums}

The formulas (11) and (12) constitute the announced spectral representation of the Polyakov loop correlator and thus, via Eq. (2), the spectral decomposition of 
the static quark-antiquark potential. The right-hand side is a double sum over all Dirac eigenvalues and we can indeed decompose the correlator into IR and UV parts by taking into account only parts of the spectrum. We now analyze numerically how the individual terms in (11) and (12) give rise to the static quark potential and how they see the QCD phase transition.

The numerical analysis is done on quenched $\mathrm{SU}(3)$ gauge configurations generated with the Lüscher-Weisz action [8] on various $L^{3} \times N$ lattices with $L=10$ and 12 and $N=4$ and 6 . All data we show here are for the largest lattice, $12^{3} \times 6$, and we have a statistics of 20 configurations both in the confined and the deconfined phase. These results were cross-checked on smaller lattices where larger statistics of $\mathcal{O}(100)$ configurations were produced. The lattice spacing was determined in [9] using a Sommer parameter of $r_{0}=0.5 \mathrm{fm}$. The gauge coupling was chosen such that we have ensembles in the confined phase at a temperature of $T=236 \mathrm{MeV}$ and ensembles in the deconfined phase at $T=412 \mathrm{MeV}$. Above $T_{c}(\sim 300 \mathrm{MeV})$ the theory has a non-vanishing Polyakov loop, which can have three possible phases. In our numerical analysis we restrict ourselves to configurations with (essentially) real Polyakov loop, since the results for ensembles with one of the two complex phases of the Polyakov loop may be related to the real case by a simple $\mathbb{Z}_{3}$ transformation. For the numerical analysis of the spectral sums (11) and (12) we computed complete sets of eigenvalues and eigenvectors of the staggered Dirac operator using ScaLAPACK routines. The results from the spectral sums were cross-checked to an evaluation of the Polyakov-loop correlators directly from the gauge links and agreement of the first 7 to 10 digits was found.

The central quantity we consider in this section is the correlator $\left\langle L(\vec{x}) L^{*}(\vec{y})\right\rangle$, evaluated with the exact formula (11). In order to improve the statistics we average over $\vec{x}$ and $\vec{y}$, keeping the distance $d$ fixed. We define

$$
C(d)=\frac{1}{3 L^{3}} \sum_{\vec{x}} \sum_{j=1,2,3}\left\langle L(\vec{x}) L^{*}\left(\vec{x}+d \widehat{e}_{j}\right)\right\rangle,
$$

where $\widehat{e}_{j}, j=1,2,3$ are the unit vectors in the three spatial directions and for $L(\vec{x}) L^{*}\left(\vec{x}+d \widehat{e}_{j}\right)$ we insert the spectral representation (11). According to Eq. (2) $C(d)$ behaves as

$$
C(d) \sim A e^{-N V(d)} .
$$

We now study the correlator $C(d)$ but sum the spectral sums in (11) only up to some largest eigenvalue $\lambda_{c u t}$. Thus for small $\lambda_{\text {cut }}$ we only see the IR contributions and as we increase $\lambda_{\text {cut }}$ towards the largest eigenvalue $\lambda_{\max }$, we gradually recover the full result. These truncated sums allow one to study how the full correlator builds up from the different contributions in the IR and UV. 


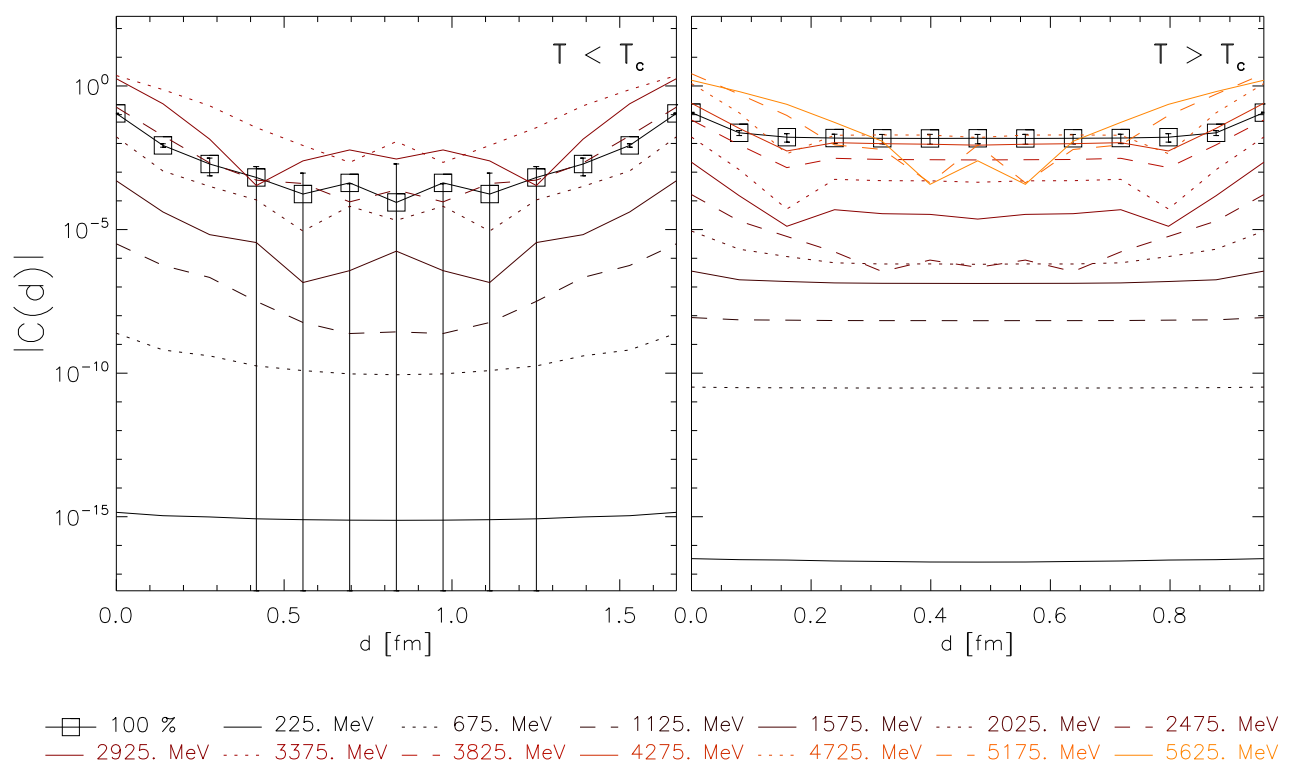

Figure 1: Absolute values of the truncated spectral sums for the Polyakov loop correlator $C(d)$ for our $12^{3} \times 6$ lattices below (lhs. plot) and above $T_{c}$ (rhs.). The symbols with error bars represent the full correlator as obtained directly from the gauge fields. The other curves are obtained from the spectral sums at various values of $\lambda_{\text {cut }}$, starting from a small cutoff for the curves at the bottom.

In Fig. 11 we show the absolute values of the truncated sums for our ensembles on $12^{3} \times 6$ lattices below (Ihs. plot) and above $T_{c}$ (rhs.). The symbols represent the data from an evaluation of the correlators $C(d)$ using directly the gauge links, and thus are the limiting values which the spectral sums approach as the cutoff $\lambda_{c u t}$ is sent towards the largest eigenvalue. Only for those we show statistical errors, which are however representative also for the truncated spectral sums. In the confined phase, the correlators show a cosh-type of behavior in $d$ which is a consequence of a non-vanishing string tension for the potential $V(d)$ in (2) and the periodic boundary conditions for the gauge fields 1 . For $T>T_{c}$ (rhs. plot) the symbols quickly form a constant plateau as is expected in the deconfined phase where the string tension vanishes.

\footnotetext{
${ }^{1}$ We remark, that the rather small lattices which we are restricted to by the need to compute the full Dirac spectrum lead to sizable statistical errors towards the minimum of the cosh. The error bars appear asymmetrically due to the logarithmic scale.
} 
The curves without symbols and error bars are the results from the spectral sums at different values of the cutoff $\lambda_{\text {cut }}$, with the curves at the bottom of the plots corresponding to a low $\lambda_{\text {cut }}$. We remark that due to the different values of the gauge coupling which we use below and above $T_{c}$, a different lattice cutoff results, such that for the ensemble below $T_{c}$ (Ihs. plot) the largest eigenvalues are near $\lambda_{\max } \sim 3500 \mathrm{MeV}$, while above $T_{c}$ (rhs.) they reach up to $\lambda_{\max } \sim 5800$ $\mathrm{MeV}$. Both phases show a similar behavior of a slow buildup of the correlator as $\lambda_{\text {cut }}$ is increased. At about $30 \%$ of the maximal eigenvalue (e.g., the $\lambda_{\text {cut }}=$ $1125 \mathrm{MeV}$ curve for the Ihs., and the $\lambda_{\text {cut }}=1575 \mathrm{MeV}$ curve on the rhs.) the shape of the correlator, cosh-type and flat, respectively, is quite visible. As $\lambda_{c u t}$ is increased further, fluctuations appear which might be a consequence of the fact that contributions from large eigenvalues are weighted much stronger. Towards the UV end, the spectral sums even overshoot the final result and settle at the exact values (symbols in the plot) only when all eigenvalues are taken into account. This overshooting phenomenon was observed already in [2] where the expectation value of a single Polyakov loop was studied.

\section{Spatial structure of the eigenvector density correlators}

Having established that the spectral sums give rise to the correct Polyakov loop correlator and carry the information about confinement versus deconfinement also when not fully summed, we now come to analyzing individual parts in the spectral sums. In particular we discuss the role of the eigenvector densities $\rho_{i}^{(k)}(\vec{x})$ in the two formulas (11) and (12).

The correlator (12) is related to the static quark potential as stated in Eq. (2). Below $T_{c}$ the theory is confining with a non-zero string tension $\sigma$, such that for sufficiently large $|\vec{x}-\vec{y}|$ we expect $\left\langle L(\vec{x}) L^{*}(\vec{y})\right\rangle \propto \exp (-N \sigma|\vec{x}-\vec{y}|)$. Above the critical temperature the confinement is gone, such that $\left\langle L(\vec{x}) L^{*}(\vec{y})\right\rangle$ is essentially constant for large $|\vec{x}-\vec{y}|$. The characteristic spatial behavior of the correlator (12) below and above $T_{c}$ can only come from the eigenvector densities $\rho_{i}^{(k)}(\vec{x})$, since their correlators are the only terms that contain a dependence on the spatial coordinates $\vec{x}$ and $\vec{y}$. Consequently the correlators of the eigenvector densities must already contain the spatial information which in the full correlator (12) gives rise to the discussed spatial behavior governed by the static potential below and above $T_{c}$.

In order to study the spatial structure of the (weighted) correlators of the eigenvector densities, we analyze

$$
C_{r, s}^{(\lambda, \mu)}(d)=\frac{1}{3 L^{3}} \sum_{\vec{x}} \sum_{j=1,2,3}\left\langle(\lambda \mu)^{N} \rho_{r}^{(\lambda)}(\vec{x}) \rho_{s}^{(\mu)}\left(\vec{x}+d \widehat{e}_{j}\right)\right\rangle .
$$


$\lambda$ and $\mu$ are eigenvalues on the positive imaginary axis and $\rho_{r}^{(\lambda)}(\vec{x}), \rho_{s}^{(\mu)}\left(\vec{x}+d \widehat{e}_{j}\right)$ are the corresponding eigenvector densities. The indices $r, s=1,2,3$ refer to our three boundary conditions $z_{1}, z_{2}, z_{3}$ as stated in (6).

The correlators (15) are rather involved objects. Beside the dependence on the distance $d$, they have four different parameters: two labels $\lambda, \mu$ for the eigenvalues and two labels $r, s$ for the 3 different boundary conditions. Thus we have to consider correlators where $\lambda=\mu$, as well as correlators with $\lambda \neq \mu$. Furthermore our formulas (11), (12) contain all possible combinations of boundary conditions. In particular in the formula for the ensemble average (12) the correlators with equal boundary conditions, $r=s$, come with a plus sign, while the correlators with differing boundary conditions, $r \neq s$, are subtracted. All these possible combinations of the parameters $\lambda, \mu, r, s$, might give rise to a different dependence of the corresponding correlators on the spatial distance d. In (12) these correlators are combined with relative signs and only this combination then gives the correct dependence of the Polyakov loop correlators on the spatial separation $d$.

The central question we want to answer in this section is whether also the individual eigenvector density correlators show a change in their spatial behavior as one crosses from the confined into the deconfined phase. A changing spatial spatial behavior of the eigenvector density correlators would be the simplest mechanism for changing the static potential from confinement to deconfinement.

While for the behavior of Dirac eigenvectors with small eigenvalues quite some information is available, very little is known about Dirac eigenvectors with eigenvalues that are not in the deep IR. Thus we here systematically study the eigenvector density correlators (15) for various combinations of the parameters $\lambda, \mu, r, s$. Since we here are predominantly interested in the decay properties of these correlators, we normalize them such that they assume the value 1 at vanishing distance $d=0$.

In Fig. 2 we begin with the simplest case, where we consider the correlation $C_{r, r}^{(\lambda, \lambda)}(d)$ of an eigenvector with itself, i.e., both densities $\rho_{r}^{(\lambda)}(\vec{x})$ in the correlator correspond to the same eigenvalue $\lambda=\mu$ and have the same boundary conditions which we set to $r=s=1$, i.e., we consider periodic boundary conditions for the Dirac operator. We remark, that the other two cases where the boundary conditions are equal, $r=s=2$ and $r=s=3$, give similar results. The Ihs. plot shows the correlator below $T_{c}$, while the rhs. is for the deconfined phase $T>T_{c}$. On the axis from the front to the back the spatial separation $d$ is plotted, while on the axis running from left to right, we plot the number of the eigenvalue $\lambda$, where at the left we start with the IR end of the spectrum 

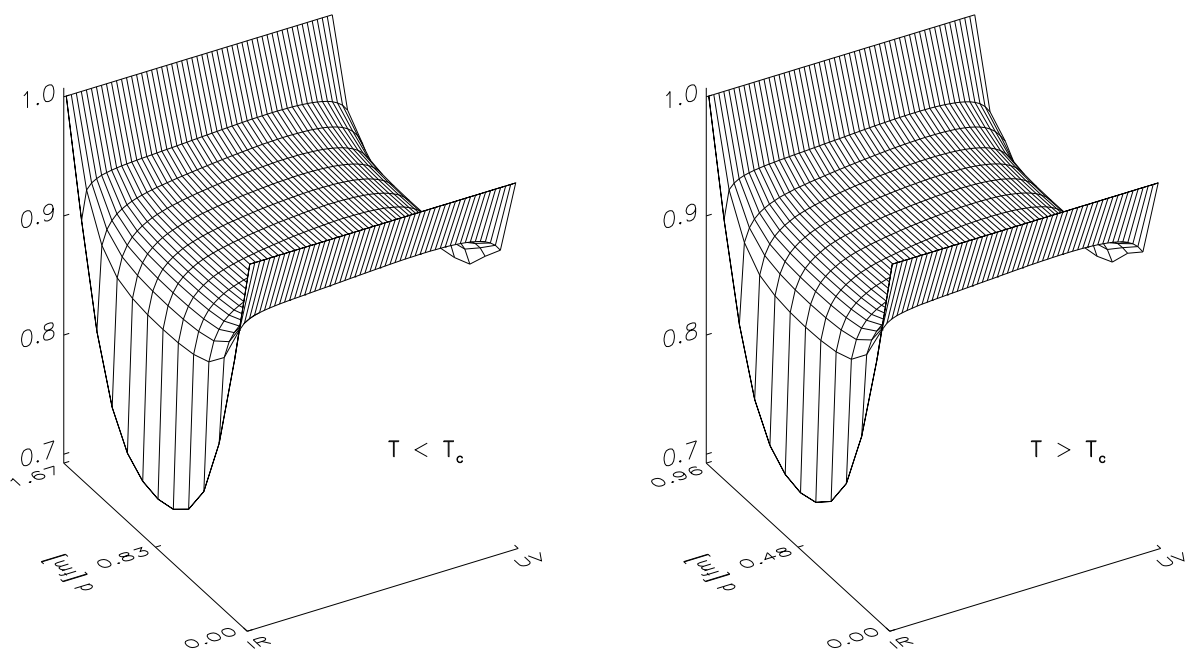

Figure 2: Normalized correlator $C_{r, r}^{(\lambda, \lambda)}(d)$ for equal eigenvalues as a function of $d$ and $\lambda$. The boundary conditions are chosen periodic, i.e., $r=1$. The lhs. plot is for $T<T_{c}$, while the rhs. is for the deconfined phase above $T_{c}$.

and the UV eigenvalues are at the right-hand side. Note that the eigenvalues are purely imaginary and each eigenvalue comes with both signs. As we have already discussed it is sufficient to sum over only one half of the eigenvalues, and in the corresponding axis of the plot we simply run through the eigenvalues on the positive imaginary axis. These eigenvalues were divided into bins, and the correlators averaged over the bins is what is shown in the 3-D mesh plot.

For both cases, $T<T_{c}$ and $T>T_{c}$ the correlator decays with $d$, which due to the periodicity gives rise to a minimum in the center followed by another increase. The decay is most prominent for the eigenvalues at the IR and UV ends of the spectrum, while near the middle of the spectrum a rather flat trough is formed. A more detailed analysis of slices through the 3-D plot reveals that at the IR and UV ends the correlator indeed gives rise to a cosh, which shows that the correlator decays exponentially. Slices through the center part of the plot show that for the bulk of the spectrum the correlators of densities with medium sized eigenvalues become flat.

However, the most remarkable finding of the plots is the fact, that the normalized density correlators below and above $T_{c}$ are almost indistinguishable. In Fig. 2 this is only a finding for a very particular correlator, namely the one where the eigenvalues of the two densities and their boundary conditions coincide, but 

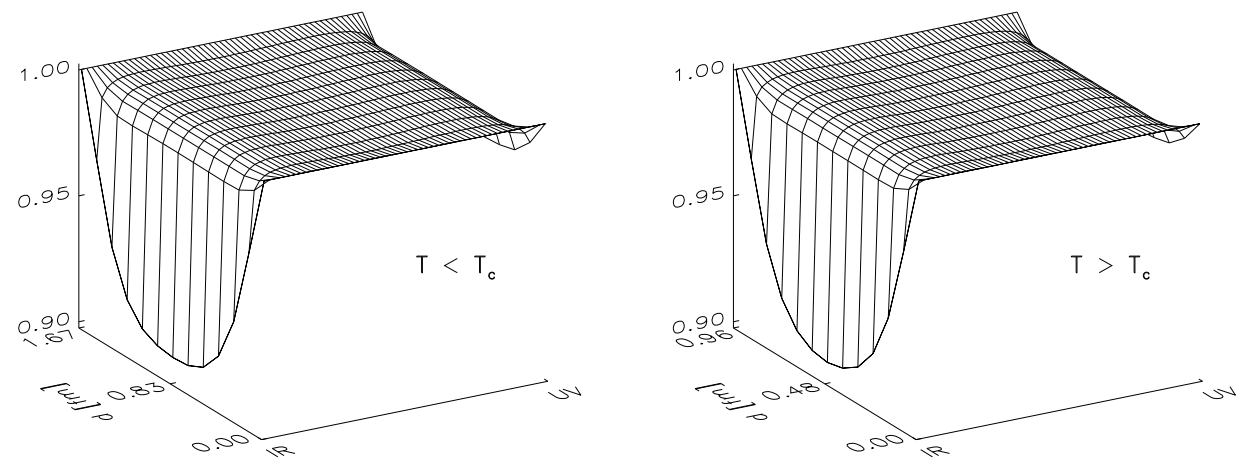

Figure 3: Same as Fig. 2, but now the correlator $C_{1,2}^{(\lambda, \lambda)}(d)$ which combines periodic conditions and the density with boundary phase $2 \pi / 3$ is shown.

we will show, that for essentially all normalized correlators contributing in (11) and (12) the difference between the confined and deconfined phases is very small.

The next correlator of densities we study is the combination where the two densities still correspond to the same eigenvalue, but have different boundary conditions. In particular we consider the combination of a periodic boundary condition and one with phase $2 \pi / 3$. The normalized correlator $C_{1,2}^{(\lambda, \lambda)}(d)$ for this choice of parameters is shown in Fig. 3. As in the case of equal boundary conditions we see that a pronounced decay is visible only for the smallest and the largest eigenvalues, while for the eigenvalues in the center of the distribution again a rather flat plateau is found. Also here the difference between the confined (Ihs. plot) and the deconfined phase (rhs.) is rather small.

So far we have restricted ourselves to correlators where the two densities were for equal eigenvalues. In Fig. 4 we now show some cases of normalized correlators of densities $C_{r, s}^{(\lambda, \mu)}(d)$ corresponding to different eigenvalues, $\lambda \neq \mu$. We always keep the eigenvalue $\mu$ fixed and plot the correlator as a function of the other eigenvalue $\lambda$ and the spatial separation $d$. For the lhs. column $\mu$ is chosen near the IR end of the spectrum, while on the rhs. $\mu$ is at the UV end. In the top row both densities are for periodic boundary conditions, while in the bottom plots periodic, combined with $2 \pi / 3$ boundary conditions are used. All plots in Fig. 4 are for $T<T_{c}$. It is obvious, that these correlators mainly give rise to a flat spatial behavior. Again the corresponding correlators for $T>T_{c}$ are almost identical.

From our analysis of the spatial behavior of $C_{r, s}^{(\lambda, \mu)}(d)$ we conclude, that the 

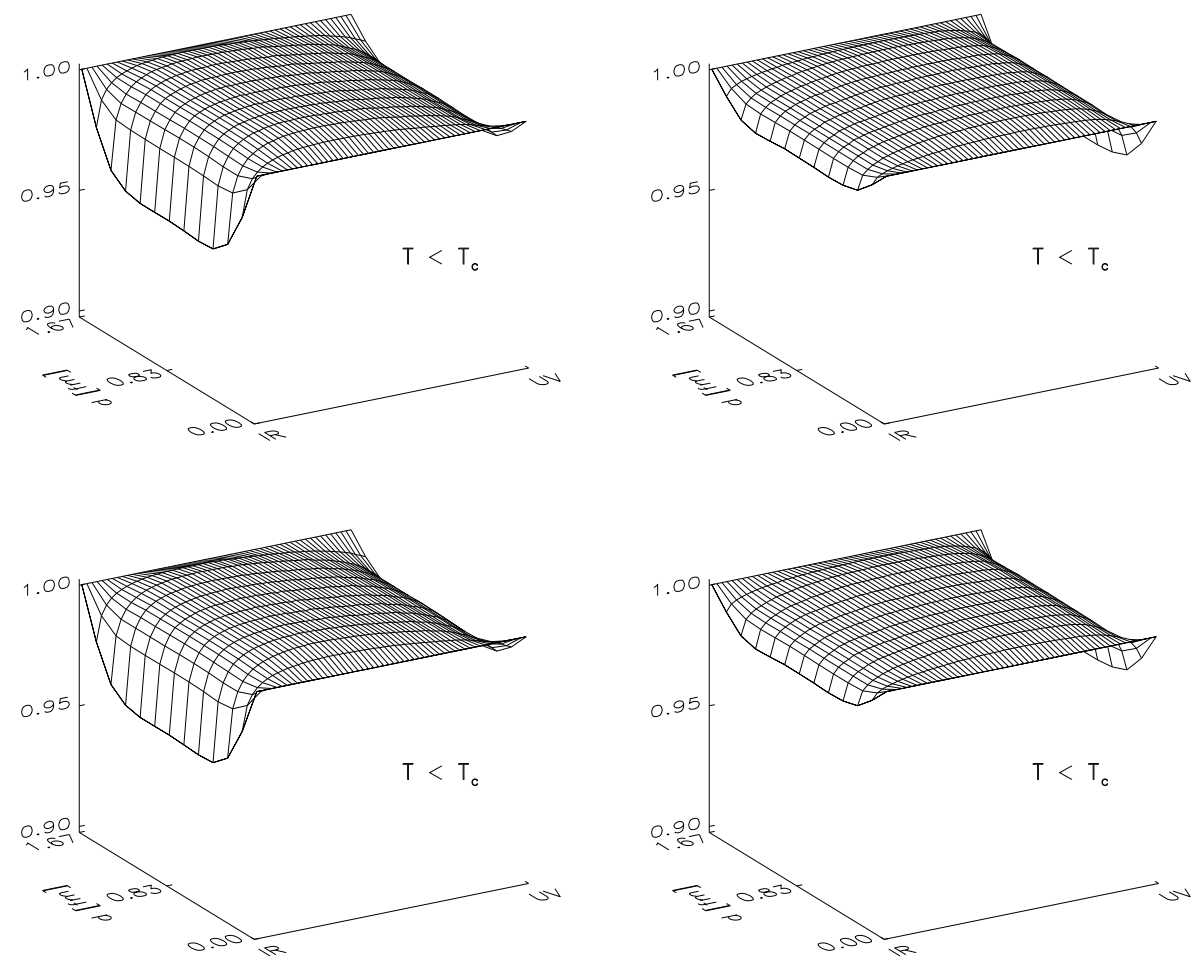

Figure 4: The normalized correlators $C_{r, s}^{(\lambda, \mu)}(d)$, where $\mu$ is held fixed at an IR value (lhs. column of plots) and at an UV value (rhs.). In the top row we show the correlator where both densities are for periodic boundary conditions, while in the bottom row, the combination of periodic with $2 \pi / 3$ boundary conditions is displayed. All plots are for $T<T_{c}$.

eigenvector density correlators (15) show a different spatial decay depending on the chosen combination of the parameters $\lambda, \mu, r$ and $s$. For some values we identify a clear exponential decay, which due to the periodicity is manifest as a cosh, while for other choices of the parameters the correlators are essentially flat. The final formula (12) for the Polyakov loop correlator contains a sum over all parameter combinations. The individual correlators then combine their specific different spatial shapes such that either the exponential decay for the confined phase, or the flat behavior of the deconfined phase emerges in the sum for the complete Polyakov loop correlator.

The most remarkable observation for the normalized eigenvector density correlators is, however, the fact that they are almost unchanged if the results for 
$T<T_{c}$ and $T>T_{c}$ are compared. We quantified this observation by analyzing the difference of the correlators $C_{r, s}^{(\lambda, \mu)}(d)$ for our ensembles below and above $T_{c}$. We found that the relative difference is below one percent throughout. The largest discrepancies (about one percent) were found at the IR and UV edges of the distributions.

We repeated the same analysis for the modified correlators

$$
\widetilde{C}_{r, s}^{(\lambda, \mu)}(d)=\frac{1}{3 L^{3}} \sum_{\vec{x}} \sum_{j=1,2,3}\left\langle\rho_{r}^{(\lambda)}(\vec{x}) \rho_{s}^{(\mu)}\left(\vec{x}+d \widehat{e}_{j}\right)\right\rangle,
$$

where the weight factor $(\lambda \mu)^{N}$ is omitted. Although these eigenvector density correlators are not directly related to the Polyakov loops, their behavior is of general interest: Hadronic correlation functions may be expressed with the spectral theorem and various correlators of Dirac eigenvectors will appear in such a representation. When crossing the phase transition, the changing hadron content might leave a trace in the correlators of the Dirac eigenvectors. In our analysis of the correlators $\widetilde{C}_{r, s}^{(\lambda, \mu)}(d)$ we found that their general behavior is very similar to the one of the weighted correlators $C_{r, s}^{(\lambda, \mu)}(d)$ as shown in Figs. $2-4$. By analyzing as before the difference of the correlators above and below $T_{c}$ we find again, that crossing the phase transition leads to changes of the correlators which are only in the one percent range.

\section{Amplitudes of the density correlators}

In the last section we studied the spatial shape of our correlators (15) and thus plotted them such that they were normalized to 1 at $d=0$. We now focus on the actual amplitudes of these correlators defined as the correlator $C_{r, s}^{(\lambda, \mu)}(0)$ taken at distance $d=0$. These amplitudes are important for understanding how the individual correlators combine in the formula (12) to build up the Polyakov loop correlation function.

In Fig. 5 we plot the amplitudes $C_{r, s}^{(\lambda, \mu)}(0)$ as a function of $\lambda$ and $\mu$ and compare different combinations of the boundary condition parameters $r$ and $s$. The plots on the lhs. are for boundary conditions $r=s=1$, and on the rhs. the combination $r=1, s=2$ is shown. The top row is for $T<T_{c}$, while at the bottom $T>T_{c}$ is shown. The plots show that the amplitudes for the contributions of different eigenvalues all have a very similar size, and only at the IR end show a sharp decrease. Furthermore the two combinations of boundary conditions ( $r=s=1$ in the lhs. plot and $r=1, s=2$ on the rhs.) give rise to almost indistinguishable amplitude distributions. The same is true for the other combinations of boundary conditions that enter in the spectral sum (12). 

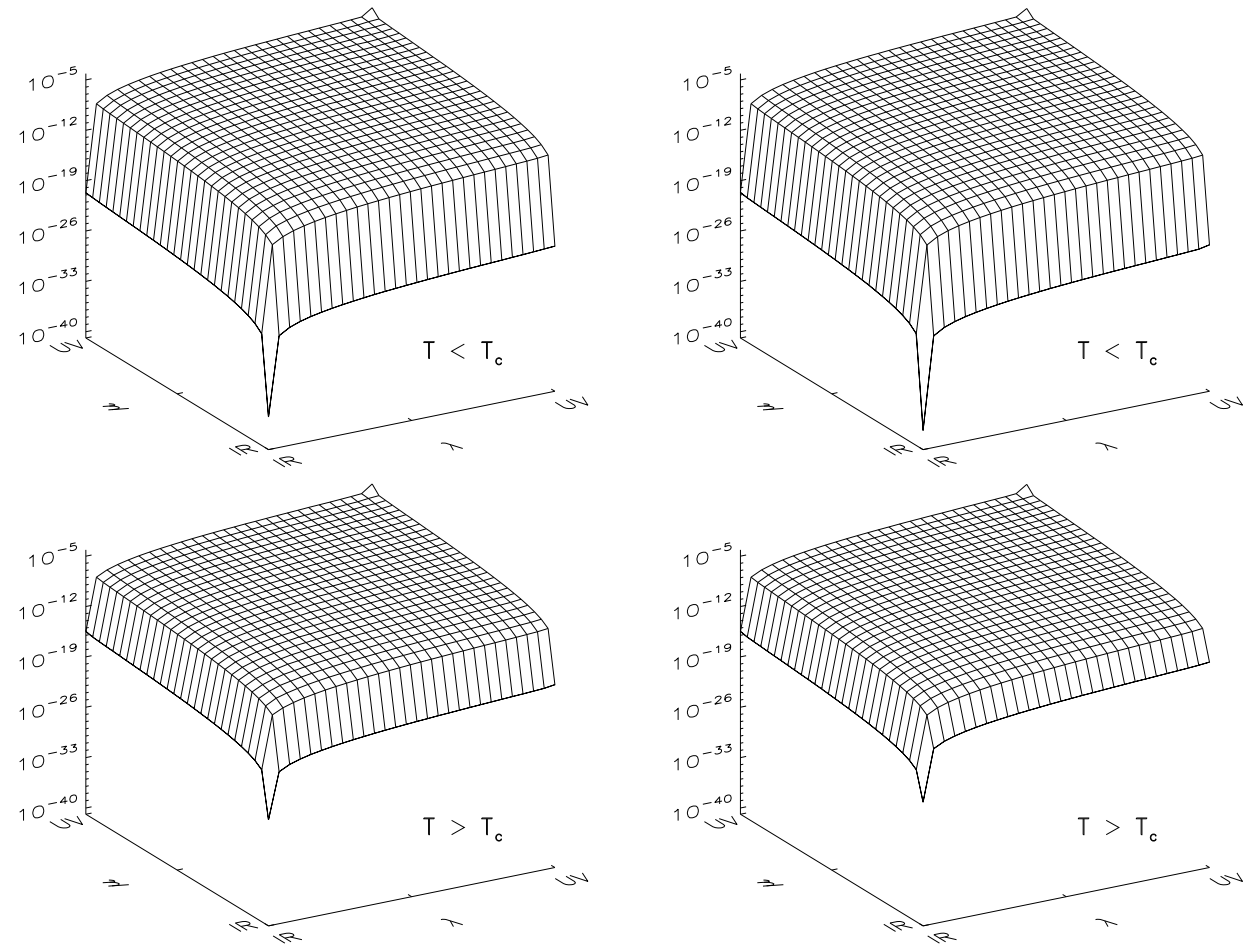

Figure 5: The amplitudes $C_{r, s}^{(\lambda, \mu)}(0)$ as a function of the eigenvalues $\lambda$ and $\mu$. The plots on the lhs. are for the boundary conditions $r=s=1$, while at the rhs. we show $r=1, s=2$. In the top row we show the amplitudes for $T<T_{c}$, while at the bottom we have $T>T_{c}$.

If we now compare the amplitudes below $T_{c}$ (top plots) to their counterparts above $T_{c}$ (bottom), we find a sizable discrepancy for some ranges of the eigenvalues $\lambda$ and $\mu$ (note that we use a logarithmic scale on the vertical axis). To illustrate this shift of the amplitudes more clearly we define the relative amplitudes

$$
\Delta_{r, s}^{(\lambda, \mu)}=\frac{\left.C_{r, s}^{(\lambda, \mu)}(0)\right|_{T<T_{c}}-\left.C_{r, s}^{(\lambda, \mu)}(0)\right|_{T>T_{c}}}{\left.C_{r, s}^{(\lambda, \mu)}(0)\right|_{T<T_{c}}+\left.C_{r, s}^{(\lambda, \mu)}(0)\right|_{T>T_{c}}}
$$

The relative amplitudes $\Delta_{r, s}^{(\lambda, \mu)}$ may assume values between -1 and 1 and are positive when the correlator amplitude is larger in the confined phase, while it is negative otherwise. For identical amplitudes one has $\Delta_{r, s}^{(\lambda, \mu)}=0$. 

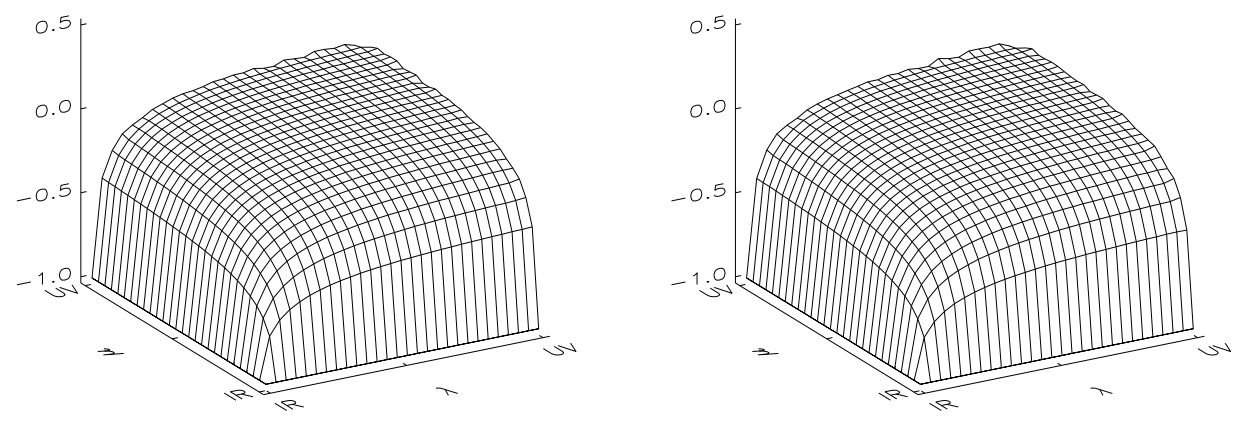

Figure 6: The relative amplitudes $\Delta_{r, s}^{(\lambda, \mu)}$ as defined in (17) as a function of $\lambda$ and $\mu$. The lhs. plot is for boundary conditions $r=s=1$, the rhs. for $r=1, s=2$.

In Fig. 6 we show the relative amplitudes $\Delta_{1,1}^{(\lambda, \mu)}$ on the lhs. and $\Delta_{1,2}^{(\lambda, \mu)}$ on the rhs. The two different combinations of boundary conditions give rise to very similar behavior. Both relative amplitudes have in common that they are negative except when both eigenvalues $\lambda$ and $\mu$ are in the upper third towards the UV end of the spectrum. This implies that the amplitudes in the IR are smaller in the confined phase, while towards the UV part of the spectrum the amplitudes are larger in that phase. Thus in the confined phase more weight is on the UV end of the spectrum, while in the deconfined phase the IR part is enhanced. This shift of the amplitudes changes the weight of eigenvector density correlators with different decay properties, which we analyzed in the last section, and in this way switches from the confining to the deconfining static potential. Since the spatial decay properties of the eigenvector density correlators are essentially invariant, it is this relative shift of the amplitudes which we identify as the mechanism giving rise to the changing behavior at $T_{c}$. 


\section{Conclusions}

In this article we have studied correlators of Polyakov loops and their spectral decomposition in terms of eigenvalues and eigenvectors of the Dirac operator. The spectral sums we discuss connect the static quark-antiquark potential to correlators of densities of the Dirac eigenvectors. From those eigenvector density correlators the static potential must build up its spatial dependence, characteristic for the confined and deconfined phases. The eigenvector density correlators were studied numerically for quenched $\mathrm{SU}(3)$ configurations below and above the transition temperature $T_{c}$.

We find that depending on the parameters (eigenvalue combination and boundary conditions), the individual eigenvector density correlators have different decay properties, ranging from a strong exponential decay to a rather flat behavior. The final Polyakov loop correlator is obtained as a combination of all these contributions and only for the full spectral sum the spatial decay properties of the Polyakov loop correlator emerge.

An interesting outcome of the numerical part of our analysis is the finding that the spatial decay properties of the (normalized) correlators of the eigenvector densities are essentially the same in the confined and the deconfined phases. The relative difference of the normalized correlators below and above $T_{c}$ was found to be less than one percent. For the amplitudes the situation is different. We show that they change when crossing $T_{c}$, such that in the deconfined phase the IR part is enhanced in the spectral sums, while the UV part is reduced. This shift of the amplitudes leads to a different combination of spatial decay properties of individual correlators and the static potential switches from confinement to deconfinement.

Having established a particular behavior of Dirac eigenvector correlators in the two phases as mechanism for shifting from confinement to deconfinement, a more systematic analysis of general eigenvector correlators appears interesting. Since with the spectral theorem hadronic observables may be expressed in terms of Dirac eigenvector correlators, a better understanding of such correlators might lead to a clearer characterization of hadronic properties in the deconfined phase.

\section{Acknowledgments}

We thank Falk Bruckmann, Christian Hagen, Christian Lang, Kurt Langfeld, Wolfgang Söldner, Pierre van Baal, Jac Verbaarschot and Andreas Wipf for discussions. The numerical analysis was done at the ZID, University of Graz. E. Bilgici is supported by the FWF Doktoratskolleg Hadrons in Vacuum, Nuclei and Stars (DK W1203-N08) and C. Gattringer by FWF Project 20330. 


\section{References}

[1] C. Gattringer, Phys. Rev. Lett. 97, 032003 (2006).

[2] F. Bruckmann, C. Gattringer, C. Hagen, Phys. Lett. B 647, 56 (2007).

[3] F. Synatschke, A. Wipf, C. Wozar, Phys. Rev. D 75, 114003 (2007).

[4] C. Hagen, F. Bruckmann, E. Bilgici, C. Gattringer, PoS(LATTICE 2007)289 [arXiv:0710.0294 [hep-lat]].

[5] W. Söldner, PoS(LATTICE2007) 222.

[6] E.Bilgici, F. Bruckmann, C. Gattringer, C. Hagen, arXiv:0801.4051 [hep-lat].

[7] F. Synatschke, A. Wipf and K. Langfeld, arXiv:0803.0271 [hep-lat].

[8] M. Lüscher, P. Weisz, Commun. Math. Phys. 97, 59 (1985); E: 98, 433 (1985); G. Curci, P. Menotti, G. Paffuti, Phys. Lett. B 130, 205 (1983); E: B 135, 516 (1984).

[9] C. Gattringer, R. Hoffmann, S. Schaefer, Phys. Rev. D 65, 094503 (2002). 